\title{
Pertuzumab for the treatment of patients with human epidermal growth factor receptor 2-positive breast cancer in Japan
}

\author{
TOMOFUMI OSAKO, REIKI NISHIMURA, YASUYUKI NISHIYAMA and MAMIKO FUJISUE \\ Department of Breast Oncology, Kumamoto Shinto General Hospital, Kumamoto, Kumamoto 862-8655, Japan \\ Received April 9, 2015; Accepted August 28, 2015
}

DOI: $10.3892 / \operatorname{mco} .2015 .640$

\begin{abstract}
Pertuzumab, a novel anti-human epidermal growth factor receptor 2 (HER2) agent, is effective for metastatic HER2-positive breast cancer when used in combination with taxane and trastuzumab. The aim of the present study was to describe the use of pertuzumab in Japan. A phase I clinical trial of pertuzumab for HER2-positive metastatic breast cancer was first conducted in the United States in 2001 (study ID no. TOC2297g) and for HER2-positive solid cancers in Japan in 2004 (study ID no. JO17076). However, Japanese patients were not enrolled in a global phase II trial for metastatic breast cancer (study ID no. BO17929) and no phase II trial of pertuzumab for Japanese patients has yet been conducted. A phase III trial on pertuzumab for metastatic breast cancer (CLEOPATRA study), which included 53 Japanese patients, revealed that pertuzumab significantly prolonged progression-free and overall survival. However, the superiority of the pertuzumab group was not verified in the subgroup analysis of Japanese patients, which was not a preplanned analysis. Therefore, a postmarketing clinical trial for Japanese patients with HER2-positive metastatic breast cancer (COMACHI study) was initiated in November, 2013, to investigate the clinical effectiveness of pertuzumab in Japanese patients. As of December, 2014, global trials on pertuzumab in the metastatic and adjuvant settings are currently ongoing. These trials included Japanese patients with HER2-positive breast cancer. Pertuzumab was approved in Japan in August, 2013 due to the positive findings of the CLEOPATRA study. Unlike the United States and Europe, the Japanes Pharmaceutical and Medical Devices Agency approved the administration of pertuzumab as second- or later-line treatment for HER2-positive metastatic breast cancer, as well as first-line treatment. Furthermore, pertuzumab may be used in combination with other chemotherapeutic agents, with the exception of docetaxel. The approval of the expanded use of pertuzumab is
\end{abstract}

Correspondence to: Dr Tomofumi Osako, Department of Breast Oncology, Kumamoto Shinto General Hospital, 1-17-27 Shinyashiki, Chuo-ku, Kumamoto, Kumamoto 862-8655, Japan

E-mail: tohsako630108@yahoo.co.jp

Key words: pertuzumab, trastuzumab, human epidermal growth factor receptor 2, breast cancer, clinical trial, Japan likely to accelerate the market penetration of pertuzumab in Japan more quickly compared with other countries.

\section{Introduction}

Human epidermal growth factor receptor 2 (HER2) is a biomarker expressed on cancer cell membranes and is overexpressed in 15-20\% of breast cancers (1-3). HER2 is a powerful predictive factor for anti-HER2 therapy, as well as a prognostic factor associated with poor clinical outcome (4-6). Trastuzumab (Herceptin), the first anti-HER2 agent, improved the clinical outcome of HER2-positive breast cancer when used in the metastatic and adjuvant settings (7-10). Lapatinib, a second-generation anti-HER2 agent, is a small molecular agent that acts on the tyrosine kinase domain of HER2. Pertuzumab and trastuzumab emtansine (T-DM1) were developed as new-generation anti-HER2 antibodies and are expected to improve the clinical outcomes of patients with HER2-positive breast cancer. Several ongoing clinical trials on these anti-HER2 agents are conducted to establish the optimal treatment strategy for HER2-positive breast cancer.

Pertuzumab blocks the domain of the HER2 protein associated with dimerization and ligand-dependently inhibits the dimerization between HER2 and other HER family members $(11,12)$. Inhibition of dimerization blocks the signaling pathway of HER2, which controls the existence and growth of cancer cells $(11,13-15)$. Similar to trastuzumab, pertuzumab has the function of antibody-dependent cell-mediated cytotoxity (16). Furthemore, pertuzumab and trastuzumab complementarily combine different domains (pertuzumab for domain II and trastuzumab for domain IV) of the HER2 protein and strongly inhibit the signaling pathway $(13,16)$.

In a phase III trial for HER2-positive metastatic breast cancer (CLEOPATRA study), the patients received placebo plus trastuzumab plus docetaxel (control group) or pertuzumab plus trastuzumab plus docetaxel (pertuzumab group) as first-line treatment until disease progression or until the development of toxicities that could not be effectively managed (13). The findings demonstrated that pertuzumab significantly prolonged progression-free survival (PFS), without increased cardiac toxicity. Therefore, in June, 2012, the US Food and Drug Administration approved the use of pertuzumab in combination with trastuzumab and docetaxel for the treatment of patients with HER2-positive metastatic breast cancer under the condition that they did not receive any prior anti-HER2 
therapy. In March, 2013, the European Medicines Agency approved pertuzumab under the same conditions. In Japan, pertuzumab was approved in August, 2013 for patients with HER2-positive metastatic breast cancer, although for reasons different than those indicated by the United States and Europe.

The aim of the present study was to describe the results of pertuzumab-related clinical trials and the specific conditions for using pertuzumab in Japan.

\section{Phase I trials}

TOC2297g study. The anti-HER2 mouse monoclonal antibody 2C4 (origin of pertuzumab) was isolated in 1990 at the same time as the isolation of the anti-HER2 mouse monoclonal antibody 4D5 (origin of trastuzumab) (17). A phase I trial of pertuzumab monotherapy for HER2-positive solid tumors (study ID no. TOC2297g) was conducted in the United States between 2001 and 2003 (18). Patients with incurable, locally advanced, recurrent or metastatic solid tumors that had progressed during or after standard therapy were recruited to a dose-escalation study of pertuzumab $(0.5-15 \mathrm{mg} / \mathrm{kg})$ administered intravenously every 3 weeks. A total of 21 patients (including 3 patients with breast cancer) received pertuzumab and 19 patients completed at least two cycles. The results demonstrated that pertuzumab was well tolerated, had a pharmacokinetic profile supporting 3-week dosing and was clinically active, which indicated that inhibition of dimerization may be an effective antitumor treatment strategy.

JO17076 study. A phase I trial including Japanese patients with HER2-positive solid tumors (study ID no. JO17076) was conducted in 2004 (19). That study evaluated the toxicity, pharmacokinetics and antitumor activity of pertuzumab in Japanese patients with HER2-positive solid tumors. Patients with solid tumors refractory to standard therapy were administered $5,10,15,20$ and $25 \mathrm{mg} / \mathrm{kg}$ pertuzumab intravenously, once every 3 weeks. A total of 18 patients with solid tumors (including 1 patient with breast cancer) were enrolled and a total of 32 cycles of pertuzumab were administered. The toxicities were generally acceptable. Grade 3 elevation of $\gamma$-glutamyl transpeptidase was observed in 1 patient at $25 \mathrm{mg} / \mathrm{kg}$ and was considered to be dose-limiting. The maximum tolerated dose level of $25 \mathrm{mg} / \mathrm{kg}$ was not reached. The serum concentration of pertuzumab slowly declined (terminal half-life of $\sim 3$ weeks). The area under the curve proportionally increased over the dose range tested. There was limited evidence of activity (stable disease, $n=2$; progressive disease, $n=13$; and non-evaluable, $n=3$ ); however, tumor shrinkage and tumor marker level decrease were observed in 1 patient with ovarian cancer and 1 patient with non-small-cell lung cancer, respectively. Pertuzumab was well tolerated up to $25 \mathrm{mg} / \mathrm{kg}$. Although objective tumor response was not observed, it is worth evaluating as a flat dose and in combination with other cytotoxic or molecular-targeted agents.

\section{Phase II trials}

BO17929 study. A phase II trial of pertuzumab and trastuzumab in patients with HER2-positive metastatic breast cancer (study ID no. BO17929) was conducted in the United States in 2006 (20). That study did not include Japanese patients. The phase II trial was a multicenter, open-label, single-arm, Simon two-stage study assessing the efficacy and safety profile of this combination in patients with HER2-positive breast cancer whose disease had progressed during prior trastuzumab-based therapy. The patients received trastuzumab weekly or every 3 weeks, and pertuzumab every 3 weeks. All 66 patients were assessable for efficacy and safety. The objective response rate was $24.2 \%$ and the clinical benefit rate was $50 \%$. A total of 5 patients $(7.6 \%)$ achieved a complete response, 11 patients (16.7\%) achieved a partial response and 17 patients $(25.8 \%)$ had stable disease. The median PFS was 5.5 months. The combination of pertuzumab and trastuzumab was well tolerated, and the adverse events were mild to moderate. Cardiac toxicity was minimal, and no patients withdrew as a result of cardiac-related adverse events. Therefore, the combination of pertuzumab and trastuzumab was effective and well tolerated in patients with HER2-positive metastatic breast cancer who experienced progression during prior trastuzumab-based therapy.

\section{Phase III trials \\ CLEOPATRA study. A phase III trial (the Clinical Evaluation} of Pertuzumab and Trastuzumab; CLEOPATRA study) was conducted for HER2-positive metastatic breast cancer as first-line treatment (Table I) (13). In that study, 808 patients with HER2-positive metastatic breast cancer were randomly assigned to receive placebo plus trastuzumab plus docetaxel (control group) or pertuzumab plus trastuzumab plus docetaxel (pertuzumab group) until disease progression or until the development of toxic effects that could not be effectively managed. The median PFS was 12.4 months in the control group and 18.5 months in the pertuzumab group [hazard ratio $(\mathrm{HR})$ for progression or death $=0.62$; $95 \%$ confidence interval $(\mathrm{CI})$ : $0.51-0.75 ; \mathrm{P}<0.001]$. The interim analysis of overall survival (OS) exhibited a strong trend in favor of pertuzumab plus trastuzumab plus docetaxel. The safety profile was generally similar between the two groups, without an increase in left ventricular systolic dysfunction. In addition, in the final assessment at 30 months of the median follow-up of the CLEOPATRA study, the median OS was 37.6 months in the control group, but had not been reached in the pertuzumab group $(\mathrm{HR}=0.66,95 \% \mathrm{CI}: 0.52-0.84 ; \mathrm{P}=0.0008)$ (21). The adverse events were similar to those reported at the primary analysis in terms of frequency, severity and specificity. Overall, these findings revealed a significant improvement in OS with pertuzumab, trastuzumab, and docetaxel in patients with HER2-positive metastatic breast cancer compared with the control group (placebo, trastuzumab and docetaxel). Furthermore, the biomarker analyses in the CLEOPATRA study revealed that HER2 was the only marker suitable for patient selection for the trastuzumab plus pertuzumab-based regimen in HER2-positive metastatic breast cancer, and that HER2, HER3 and PIK3CA were relevant prognostic factors (22).

Moreover, the results demonstrated that Asian patients exhibited a lower tolerability of docetaxel, despite the fact that the survival benefits were comparable between regions (23). Docetaxel dose reductions to $<75 \mathrm{mg} / \mathrm{m}^{2}$ were more common in Asian patients compared with patients from other regions, and docetaxel dose escalations to $100 \mathrm{mg} / \mathrm{m}^{2}$ were less frequent in Asian patients compared with patients from other regions. The incidence rates of edema, myalgia, nail disorders, febrile 
Table I. Phase III studies on pertuzumab.

\begin{tabular}{|c|c|c|c|c|}
\hline Study name & Treatment setting & Study arms & Enrollment no. & Outcome \\
\hline CLEOPATRA & Metastatic & $\begin{array}{l}\text { Placebo+T+DTX } \\
\text { P+T+DTX }\end{array}$ & $\begin{array}{l}404 \\
402\end{array}$ & $\begin{array}{l}\text { PFS, } 12.4 \text { months } \\
\text { PFS, } 18.5 \text { months }\end{array}$ \\
\hline MARIANNE & Metastatic & $\begin{array}{l}\text { T+DTX or PTX } \\
\text { Placebo+T-DM1 } \\
\text { P+T-DM1 }\end{array}$ & $\begin{array}{c}1,095 \\
\text { (total, estimated) }\end{array}$ & $\mathrm{NS}$ \\
\hline PERUSE & Metastatic & $\mathrm{P}+\mathrm{T}+\operatorname{tax} a n e$ & $\begin{array}{c}1,500 \\
\text { (total, estimated) }\end{array}$ & Recruiting $^{\mathrm{a}}$ \\
\hline PHEREXA & Metastatic & $\begin{array}{l}\mathrm{T}+\mathrm{X} \\
\mathrm{P}+\mathrm{T}+\mathrm{X}\end{array}$ & $\begin{array}{c}452 \\
\text { (total, estimated) }\end{array}$ & Awaiting $^{\mathrm{b}}$ \\
\hline APHINITY & Adjuvant & $\begin{array}{l}\text { Placebo+T+chemotherapy } \\
\mathrm{P}+\mathrm{T}+\text { chemotherapy }\end{array}$ & $\begin{array}{c}4,808 \\
\text { (total, estimated) }\end{array}$ & Awaiting $^{\mathrm{b}}$ \\
\hline KAITLIN & Adjuvant & $\begin{array}{l}\mathrm{A} \rightarrow \mathrm{P}+\mathrm{T}+\operatorname{tax} a n e \\
\mathrm{~A} \rightarrow \mathrm{P}+\mathrm{T}-\mathrm{DM} 1\end{array}$ & $\begin{array}{c}2,500 \\
\text { (total, estimated) }\end{array}$ & Recruiting $^{\mathrm{a}}$ \\
\hline
\end{tabular}

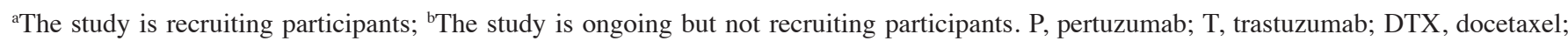
PTX, paclitaxel; X, capecitabine; T-DM1, trastuzumab emtansine; A, anthracycline-based chemotherapy; NS, no significant difference (only in a press release); PFS, progression-free survival.

neutropenia, upper respiratory tract infections, decreased appetite and skin rash, were at least twice as high in Asian patients compared with patients from other regions. However, the adverse events did not result in a reduction in the median number of study treatment cycles administered to Asian patients. Efficacy analyses per region revealed HRs similar to those of the whole intention-to-treat population for PFS and OS.

A total of 53 Japanese patients were enrolled in the CLEOPATRA study. A subgroup analysis of these 53 patients was performed (not intended) to meet the requirement of the Japanese Pharmaceutical and Medical Devices Agency (PMDA) for drug approval in Japan. However, the superiority of pertuzumab was not verified in terms of the PFS and OS of Japanese patients (24). The reason for the discrepancy in the clinical outcome among regions is unclear. One possible explanation may be that the baseline characteristics of the Japanese patients between the pertuzumab group and the control group were not well-balanced due to the limited number of the subgroup patients. The pertuzumab group included more patients with visceral diseases or with HER2 $\left(2^{+}\right)$among the Japanese patients of the CLEOPATRA study (data not shown). Therefore, an additional postmarketing clinical study was planned to confirm the efficacy and safety of pertuzumab combined with trastuzumab and docetaxel as first-line treatment in Japanese patients with HER2-positive inoperable or recurrent breast cancer (details described below) (24).

\section{Neoadjuvant setting}

NEOSPHERE study. The neoadjuvant study of pertuzumab and trastuzumab in an early regimen evaluation (NEOSPHERE) is a randomised, multicenter, international phase II study that was conducted on 417 patients with newly diagnosed HER2-positive, locally advanced, inflammatory or early-stage breast cancer (25). Japanese patients were not enrolled in that study. The participants were randomly assigned to four study arms and received 4 cycles (12 weeks) of neoadjuvant treatment: Trastuzumab $(8 \mathrm{mg} / \mathrm{kg}$ loading dose, followed by $6 \mathrm{mg} / \mathrm{kg}$ every 3 weeks) plus docetaxel $\left(75 \mathrm{mg} / \mathrm{m}^{2}\right.$, escalating, if tolerated, to $100 \mathrm{mg} / \mathrm{m}^{2}$ every 3 weeks) (group A); pertuzumab (loading dose $840 \mathrm{mg}$, followed by $420 \mathrm{mg}$ every 3 weeks) and trastuzumab plus docetaxel (group B); pertuzumab and trastuzumab (group C); and pertuzumab plus docetaxel (group D). The primary endpoint was pathological complete response. The secondary endpoints included clinical response, time-to-clinical response, safety profile, disease-free survival (DFS), breast-conserving surgery rate and biomarker assessment. The results of the study demonstrated that group B exhibited a significant improvement in the pathological complete response rate compared with the patients administered trastuzumab plus docetaxel (no significant differences in tolerability). The tumors in a proportion of the women in group $\mathrm{C}$ were eradicated, with a favorable safety profile.

TRYPHAENA study. The tolerability of pertuzumab, trastuzumab and anthracyclines in neoadjuvant breast cancer treatment (TRYPHAENA) is a randomized, multicenter phase II study that was conducted on 225 patients with HER2-positive, locally advanced, inflammatory, or early-stage breast cancer, with tumors sized $>2 \mathrm{~cm}$ (26). Japanese patients were not enrolled in that study. The patients were randomly assigned to one of three neoadjuvant pertuzumab regimens: Arm A (3 cycles of 5-fluorouracil, epirubicin and cyclophosphamide plus trastuzumab plus pertuzumab, followed by 3 cycles of docetaxel plus trastuzumab plus pertuzumab); Arm B (3 cycles of 5-fluorouracil, epirubicin and cyclophosphamide, followed by 3 cycles of docetaxel plus trastuzumab plus pertuzumab); and Arm C (6 cycles of docetaxel plus carboplatin plus trastuzumab plus pertuzumab). The primary endpoint was cardiac safety. The secondary endpoints included pathological complete response, clinical response, breast-conserving surgery rate, DFS, PFS, OS and 
biomarker assessment. The results of the study were as follows: The rates of total pathological complete response in the three arms were 56.2 (Arm A), 54.7 (Arm B) and 63.6\% (Arm C). Moreover, no new or unexpected cardiac or other adverse events were observed in any of the study arms. The adverse events observed were consistent with those reported in previous studies of pertuzumab, trastuzumab and chemotherapy, either in combination or alone.

\section{Ongoing trials (metastatic setting)}

MARIANNE study. T-DM1 plus pertuzumab/pertuzumab-placebo vs. trastuzumab plus a taxane in patients with metastatic breast cancer (MARIANNE, NCT01120184) is a randomized, three-arm, multicenter, phase III study conducted to evaluate the efficacy and safety of T-DM1 with pertuzumab or T-DM1 with pertuzumab-placebo (blinded for pertuzumab) vs. the combination of trastuzumab plus a taxane (docetaxel or paclitaxel) in patients with HER2-positive progressive or recurrent locally advanced or previously untreated metastatic breast cancer (Table I) (27). The primary endpoints were PFS based on tumor assessments performed by an independent review facility, and the incidence of adverse events. Japanese patients were enrolled in this study.

F. Hoffmann-La Roche Ltd. (Basel, Switzerland) announced the first results of the MARIANNE study in a press release on the internet on December 19, 2014 (28). The three study arms exhibited similar PFS and the study did not meet the PFS superiority endpoint for T-DM1-containing regimens.

PERUSE study. Pertuzumab in combination with trastuzumab and a taxane as first-line treatment in patients with HER2-positive advanced breast cancer (PERUSE study ID no. NCT01572038) is a multicenter, open-label, single-arm phase IIIb study conducted to evaluate the safety and tolerability of pertuzumab in combination with trastuzumab and a taxane as first-line treatment in patients with metastatic or locally recurrent HER2-positive breast cancer (29). The patients are scheduled to receive pertuzumab and trastuzumab plus a taxane every 3 weeks. The taxanes are selected among docetaxel, paclitaxel, or nab-paclitaxel. The anticipated time of study treatment is until disease progression or until the occurrence of unacceptable toxicity. This study is currently recruiting participants.

VELVET study. Pertuzumab in combination with trastuzumab and vinorelbine as first-line treatment in patients with metastatic or locally advanced HER2-positive breast cancer (VELVET study ID no. NCT01565083) is a two-cohort, open-label, multicenter, phase II study conducted to assess the safety and efficacy of pertuzumab administered in combination with trastuzumab and vinorelbine as first-line treatment in patients with metastatic or locally advanced HER2-positive breast cancer (30). The patients are scheduled to receive $840 \mathrm{mg}$ of pertuzumab and $8 \mathrm{mg} / \mathrm{kg}$ of trastuzumab administered sequentially as separate intravenous infusions on day 1 and 2, respectively (cycle 1 ). From cycle 2 onwards, the patients will receive $420 \mathrm{mg}$ of pertuzumab and $6 \mathrm{mg} / \mathrm{kg}$ of trastuzumab, administered either sequentially as separate intravenous infusions on day 1 and day 1 or 2 , respectively (cohort 1), or together in one infusion bag on day 1 (cohort 2) every 3 weeks. Vinorelbine will be administered at $25 \mathrm{mg} / \mathrm{m}^{2}$ on days 2 and 9 of cycle 1 , and at $30-35 \mathrm{mg} / \mathrm{m}^{2}$ on days 1 and 8 (or days 2 and 9) of each following 3-week cycle. The anticipated time of study treatment is until disease progression, until the occurrence of unacceptable toxicity, or until withdrawal of consent or death. The primary endpoint is overall response rates assessed by the investigator. This study is currently recruiting participants.

PHEREXA study. Combination of trastuzumab and capecitabine with or without pertuzumab in patients with HER2-positive metastatic breast cancer (PHEREXA study ID no. NCT01026142) is a randomized, open-label, two-arm, phase III study conducted to evaluate the efficacy and safety of a combination of trastuzumab and capecitabine, with or without pertuzumab, in patients with HER2-positive metastatic breast cancer (Table I) (31). Eligibility requires progression during or following previous trastuzumab therapy for metastatic disease. The study treatment is to continue until disease progression or until the occurrence of unacceptable toxicity. The primary endpoint is PFS (independent assessment). This study is ongoing, but is not recruiting participants. Japanese patients were not enrolled in this study.

PERTAIN study. Pertuzumab in combination with trastuzumab plus an aromatase inhibitor in patients with hormone receptor-positive HER2-positive metastatic breast cancer (PERTAIN study ID no. NCT01491737) is a randomized, open-label, two-arm, multicenter, phase II study conducted to evaluate the efficacy and safety of pertuzumab in combination with trastuzumab plus an aromatase inhibitor in the first-line treatment of patients with HER2-positive and hormone receptor-positive advanced breast cancer (32). The patients will be randomized to one of two treatment arms: Pertuzumab plus trastuzumab plus aromatase inhibitor (Arm 1); or trastuzumab plus aromatase inhibitor (Arm 2). The patients may also receive induction chemotherapy (a taxane, either docetaxel or paclitaxel) at the investigator's discretion. The anticipated time of the study treatment is until disease progression or until occurrence of unacceptable toxicity. The primary endpoint is PFS. This study is currently recruiting participants.

$J B C R G-M 03$ study. Eribulin in combination with trastuzumab and pertuzumab for advanced or recurrent HER2-positive breast cancer (JBCRG-M03 and UMIN000012232) is a multicenter, single-arm, phase II trial conducted in Japan to evaluate the usefulness of eribulin in combination with trastuzumab and pertuzumab as first- or second-line therapy for patients with advanced or recurrent HER2-positive breast cancer (Table II) (33). The first endpoint is PFS and the secondary endpoints are response rate, safety, OS, efficacy following prior use of pertuzumab, compliance to eribulin and the efficacy of later therapy. This study is currently recruiting participants (33).

COMACHI study. Chugai Pharmaceutical Co., Ltd. (Kamakura, Japan) designed an additional postmarketing clinical study to confirm the superiority of the efficacy of pertuzumab combined with trastuzumab and docetaxel compared with the control group of the CLEOPATRA study (Combination of Monoclonal Antibodies for Comprehensive HER2 dimerization Inhibition; COMACHI study ID no. JO29186) (Table II) (34). This study is a multicenter, single-arm trial. The eligibility and exclusion criteria for this study were almost identical to those for the CLEOPATRA study (20). The primary endpoint is PFS and the secondary endpoints are OS, objective response rate, duration of 
Table II. Clinical studies of pertuzumab in Japan.

\begin{tabular}{llcccc}
\hline Study name & Treatment setting & Study phase & Study arms & Enrollment no. & Outcome \\
\hline JO17076 & Metastatic & I & P & 18 (1 breast cancer) & $\begin{array}{l}\text { Tolerated up } \\
\text { to } 25 \mathrm{mg} / \mathrm{kg}^{\mathrm{a}}\end{array}$ \\
JBCRG-M03 & $\begin{array}{l}\text { Metastatic, first- } \\
\text { or second-line } \\
\text { Metastatic, first-line }\end{array}$ & II & Eribulin+T+P & 48 (total, estimated) & Recruiting $^{\mathrm{a}}$ \\
COMACHI & IV & P+T+DTX & 130 (total, estimated) & Recruiting $^{\mathrm{a}}$ \\
\hline
\end{tabular}

${ }^{a}$ The study is recruiting participants. P, pertuzumab; T, trastuzumab; DTX, docetaxel.

objective response and safety. The target sample size of this study is 130 patients. This study is currently recruiting participants. The duration of recruitment for this trial is from November, 2013 to October, 2015.

\section{Ongoing trials (adjuvant setting)}

APHINITY study. Pertuzumab in addition to chemotherapy and trastuzumab as adjuvant therapy in patients with HER2-positive primary breast cancer (APHINITY study ID no. NCT01358877) is a randomized, double-blind, placebo-controlled, two-arm, phase III study conducted to assess the safety and efficacy of pertuzumab in addition to chemotherapy plus trastuzumab as adjuvant therapy in patients with operable HER2-positive primary breast cancer (Table I) (35). The patients will be postoperatively randomized to receive either pertuzumab or a placebo every 3 weeks for 1 year, in addition to 6-8 cycles of chemotherapy and 1 year of trastuzumab administered every 3 weeks. The anticipated time of the study treatment is 52 weeks. The primary endpoint is invasive DFS (IDFS). This study is ongoing, but is not recruiting participants. Japanese patients were enrolled in this study.

KAITLIN study. T-DM1 (Kadcyla) plus pertuzumab (Perjeta) following anthracyclines in comparison with trastuzumab plus pertuzumab and a taxane following anthracyclines as adjuvant therapy in patients with operable HER2-positive primary breast cancer (KAITLIN study ID no. NCT01966471) is a two-arm, randomized, open-label, multicenter, phase III study conducted to evaluate the efficacy and safety of T-DM1 in combination with pertuzumab vs. trastuzumab in combination with pertuzumab and a taxane as adjuvant therapy in patients with HER2-positive primary invasive breast cancer (Table I) (36). Following surgery and anthracycline-based chemotherapy, the patients will receive either $3.6 \mathrm{mg} / \mathrm{kg}$ of T-DM1 and $420 \mathrm{mg}$ of pertuzumab every 3 weeks or $6 \mathrm{mg} / \mathrm{kg}$ of trastuzumab every 3 weeks in combination with pertuzumab and a taxane. The anticipated time of HER2-targeted study treatment is $\leq 1$ year. The primary endpoint is IDFS, which is defined as the time from randomization to occurrence of ipsilateral breast cancer recurrence, second primary invasive breast cancer, distant recurrence, or death by any cause. The secondary endpoints are IDFS plus second primary non-breast cancer, excluding non-melanoma skin cancers and carcinoma in situ of any site, DFS (time between randomization and first occurrence of IDFS, second primary non-breast cancer and contralateral or ipsilateral ductal carcinoma in situ), distant recurrence-free interval (time between randomization and first occurrence of distant breast cancer recurrence), OS and incidence of adverse events. This study is currently recruiting participants. Japanese patients were enrolled in this study.

Special status in Japan. The unique circumstances related to pertuzumab treatment in Japan differ from those in other countries. First, the PMDA allowed the use of pertuzumab not only as first-line, but also as second- or later-line treatment for patients with HER2-positive metastatic breast cancer. Moreover, Japanese physicians may use pertuzumab for patients who previously received trastuzumab treatment in a metastatic setting. Therefore, the market penetration of pertuzumab in Japan may be quicker compared with that in other countries.

Second, the PMDA did not limit the chemotherapeutic agents to be administered with pertuzumab and trastuzumab. The Japanese package insert states that the results of the CLEOPATRA study should be referred to when the chemotherapeutic agents are selected. Japanese physicians may use chemotherapeutic agents other than docetaxel (i.e., paclitaxel or eriburin) in clinical practice. As mentioned above, the study of eribulin in combination with trastuzumab and pertuzumab for advanced or recurrent HER2-positive breast cancer (JBCRG-M03 study) has been initiated at several institutions in Japan.

Finally, the approval of T-DM1 in September 2013, immediately following the approval of pertuzumab by the PMDA, disrupted the treatment of patients with HER2-positive metastatic breast cancer in Japan. Similar to pertuzumab, the PMDA allowed the use of T-DM1 for all-line treatment of patients with HER2-positive metastatic breast cancer, although the Japanese T-DM1 package insert indicated that T-DM1 should be used for patients who previously received trastuzumab and taxane, based on the results of the EMILIA study (37). The majority of Japanese physicians tend to select pertuzumab-based regimens as first-line treatment followed by T-DM1, according to several guidelines; however, the reverse sequence of administration of pertuzumab regimens and T-DM1 is not prohibited. Furthermore, the appropriate sequence of HER2-directed therapy, including lapatinib regimens, trastuzumab regimens and T-DM1 for patients who had prior trastuzumab-based therapies, remains unclear.

In conclusion, we reviewed certain clinical trials on pertuzumab and outlined the special characteristics of pertuzumab 
use in Japan. Pertuzumab and T-DM1 may be used in Japan for patients with HER2-positive metastatic breast cancer irrespective of the treatment history, and chemotherapeutic agents other than docetaxel may be selected to be combined with pertuzumab. This may be beneficial for Japanese patients; however, without clear evidence of the efficacy of pertuzumab, there may be some confusion in the treatment strategy of HER2-positive metastatic breast cancer. Hereafter, new treatment strategies must be developed for HER2-positive breast cancer patients through clinical trials or through comprehensive data collection in clinical practice in Japan.

\section{References}

1. Slamon DJ, Clark GM, Wong SG, Levin WJ, Ullrich A and McGuire WL: Human breast cancer: Correlation of relapse and survival with amplification of the HER-2/neu oncogene. Science 235: 177-182, 1987.

2. Slamon DJ, Godolphin W, Jones LA, Holt JA, Wong SG, Keith DE, Levin WJ, Stuart SG, Udove J, Ullrich A, et al: Studies of the HER-2/neu proto-oncogene in human breast and ovarian cancer. Science 244: 707-712, 1989.

3. Gusterson BA, Gelber RD, Goldhirsch A, Price KN, Säve-Söderborgh J, Anbazhagan R, Styles J, Rudenstam CM, Golouh R, Reed R, et al; International (Ludwig) Breast Cancer Study Group: Prognostic importance of c-erbB-2 expression in breast cancer. J Clin Oncol 10: 1049-1056, 1992.

4. Seshadri R, Firgaira FA, Horsfall DJ, McCaul K, Setlur V and Kitchen P; The South Australian Breast Cancer Study Group: Clinical significance of HER-2/neu oncogene amplification in primary breast cancer. J Clin Oncol 11: 1936-1942, 1993.

5. Ravdin PM and Chamness GC: The c-erbB-2 proto-oncogene as a prognostic and predictive marker in breast cancer: A paradigm for the development of other macromolecular markers - a review. Gene 159: 19-27, 1995.

6. Press MF, Bernstein L, Thomas PA, Meisner LF, Zhou JY, Ma Y, Hung G, Robinson RA, Harris C, El-Naggar A, et al: HER-2/neu gene amplification characterized by fluorescence in situ hybridization: Poor prognosis in node-negative breast carcinomas. J Clin Oncol 15: 2894-2904, 1997.

7. Slamon DJ, Leyland-Jones B, Shak S, Fuchs H, Paton V, Bajamonde A, Fleming T, Eiermann W, Wolter J, Pegram M, et al: Use of chemotherapy plus a monoclonal antibody against HER2 for metastatic breast cancer that overexpresses HER2. N Engl J Med 344: 783-792, 2001

8. Piccart-Gebhart MJ, Procter M, Leyland-Jones B, Goldhirsch A, Untch M, Smith I, Gianni L, Baselga J, Bell R, Jackisch C, et al; Herceptin Adjuvant (HERA) Trial Study Team: Trastuzumab after adjuvant chemotherapy in HER2-positive breast cancer. N Engl J Med 353: 1659-1672, 2005

9. Gianni L, Dafni U, Gelber RD, Azambuja E, Muehlbauer S, Goldhirsch A, Untch M, Smith I, Baselga J, Jackisch C, et al; Herceptin Adjuvant (HERA) Trial Study Team: Treatment with trastuzumab for 1 year after adjuvant chemotherapy in patients with HER2-positive early breast cancer: A 4-year follow-up of a randomised controlled trial. Lancet Oncol 12: 236-244, 2011.

10. Goldhirsch A, Gelber RD, Piccart-Gebhart MJ, de Azambuja E, Procter M, Suter TM, Jackisch C, Cameron D, Weber HA, Heinzmann D, et al; Herceptin Adjuvant (HERA) Trial Study Team: 2 years versus 1 year of adjuvant trastuzumab for HER2-positive breast cancer (HERA): An open-label, randomised controlled trial. Lancet 382: 1021-1028, 2013.

11. Agus DB, Akita RW, Fox WD, Lewis GD, Higgins B, Pisacane PI, Lofgren JA, Tindell C, Evans DP, Maiese K, et al: Targeting ligand-activated ErbB2 signaling inhibits breast and prostate tumor growth. Cancer Cell 2: 127-137, 2002.

12. Hughes JB, Berger C, Rødland MS, Hasmann M, Stang E and Madshus IH: Pertuzumab increases epidermal growth factor receptor down-regulation by counteracting epidermal growth factor receptor-ErbB2 heterodimerization. Mol Cancer Ther 8: 1885-1892, 2009.

13. Baselga J, Cortés J, Kim SB, Im SA, Hegg R, Im YH, Roman L, Pedrini JL, Pienkowski T, Knott A, et al; CLEOPATRA Study Group: Pertuzumab plus trastuzumab plus docetaxel for metastatic breast cancer. N Engl J Med 366: 109-119, 2012.
14. Citri A, Skaria KB and Yarden Y: The deaf and the dumb: The biology of ErbB-2 and ErbB-3. Exp Cell Res 284: 54-65, 2003.

15. Franklin MC, Carey KD, Vajdos FF, Leahy DJ, de Vos AM and Sliwkowski MX: Insights into ErbB signaling from the structure of the ErbB2-pertuzumab complex. Cancer Cell 5: 317-328, 2004.

16. Scheuer W, Friess T, Burtscher H, Bossenmaier B, Endl J and Hasmann M: Strongly enhanced antitumor activity of trastuzumab and pertuzumab combination treatment on HER2-positive human xenograft tumor models. Cancer Res 69: 9330-9336, 2009.

17. Fendly BM, Winget M, Hudziak RM, Lipari MT, Napier MA and Ullrich A: Characterization of murine monoclonal antibodies reactive to either the human epidermal growth factor receptor or HER2/neu gene product. Cancer Res 50: 1550-1558, 1990.

18. Agus DB, Gordon MS, Taylor C, Natale RB, Karlan B, Mendelson DS, Press MF, Allison DE, Sliwkowski MX, Lieberman G, et al: Phase I clinical study of pertuzumab, a novel HER dimerization inhibitor, in patients with advanced cancer. J Clin Oncol 23: 2534-2543, 2005.

19. Yamamoto N, Yamada Y, Fujiwara Y, Yamada K, Fujisaka Y, Shimizu T and Tamura T: Phase I and pharmacokinetic study of HER2-targeted rhuMAb 2C4 (pertuzumab, RO4368451) in Japanese patients with solid tumors. Jpn J Clin Oncol 39: 260-266, 2009.

20. Baselga J, Gelmon KA, Verma S, Wardley A, Conte P, Miles D, Bianchi G, Cortes J, McNally VA, Ross GA, et al: Phase II trial of pertuzumab and trastuzumab in patients with human epidermal growth factor receptor 2-positive metastatic breast cancer that progressed during prior trastuzumab therapy. J Clin Oncol 28: 1138-1144, 2010.

21. Swain SM, Kim SB, Cortés J, Ro J, Semiglazov V, Campone M, Ciruelos E, Ferrero JM, Schneeweiss A, Knott A, et al: Pertuzumab, trastuzumab, and docetaxel for HER2-positive metastatic breast cancer (CLEOPATRA study): Overall survival results from a randomised, double-blind, placebo-controlled, phase 3 study. Lancet Oncol 14: 461-471, 2013.

22. Baselga J, Cortés J, Im SA, Clark E, Ross G, Kiermaier A and Swain SM: Biomarker analyses in CLEOPATRA: A phase III, placebo-controlled study of pertuzumab in human epidermal growth factor receptor 2-positive, first-line metastatic breast cancer. J Clin Oncol 32: 3753-3761, 2014.

23. Swain SM, Im YH, Im SA, Chan V, Miles D, Knott A, Clark E, Ross G and Baselga J: Safety profile of pertuzumab with trastuzumab and docetaxel in patients from Asia with human epidermal growth factor receptor 2-positive metastatic breast cancer: Results from the phase III trial CLEOPATRA. Oncologist 19: 693-701, 2014.

24. Pharmaceuticals and Medical Devices Agency: Report on the deliberation results of Parjeta 2013. https://www.pmda. go.jp/files/000153631.pdf. Accessed April 2, 2015.

25. Gianni L, Pienkowski T, Im YH, Roman L, Tseng LM, Liu MC, Lluch A, Staroslawska E, de la Haba-Rodriguez J, Im SA, et al: Efficacy and safety of neoadjuvant pertuzumab and trastuzumab in women with locally advanced, inflammatory, or early HER2-positive breast cancer (NeoSphere): A randomised multicentre, open-label, phase 2 trial. Lancet Oncol 13: 25-32, 2012.

26. Schneeweiss A, Chia S, Hickish T, Harvey V, Eniu A, Hegg R, Tausch C, Seo JH, Tsai YF, Ratnayake J, et al: Pertuzumab plus trastuzumab in combination with standard neoadjuvant anthracycline-containing and anthracycline-free chemotherapy regimens in patients with HER2-positive early breast cancer: A randomized phase II cardiac safety study (TRYPHAENA). Ann Oncol 24: 2278-2284, 2013

27. ClinicalTrials.gov: A study of trastuzumab emtansine (T-DM1) plus pertuzumab/pertuzumab placebo versus trastuzumab [Herceptin] plus a taxane in patients with metastatic breast cancer (MARIANNE). https://clinicaltrials.gov/ct2/show/NCT01120184. Accessed April 2, 2015.

28. F. Hoffmann-La Roche Ltd.: Update on phase III MARIANNE study in people with previously untreated advanced HER2-positive breast cancer (media release, Basel, 19 December 2014). http://www.roche.com/media/store/releases/med-cor2014-12-19.htm. Accessed April 2, 2015.

29. ClinicalTrials.gov: A study of pertuzumab in combination with Herceptin (trastuzumab) and a taxane in first-line treatment in patients with HER2-positive advanced breast cancer (PERUSE). https://clinicaltrials.gov/ct2/show/NCT01572038. Accessed April 2, 2015. 
30. ClinicalTrials.gov: A study of pertuzumab in combination with Herceptin (trastuzumab) and vinorelbine in first line in patients with metastatic or locally advanced HER2-positive breast cancer. https://clinicaltrials.gov/ct2/show/NCT01565083. Accessed April 2, 2015.

31. ClinicalTrials.gov: A study of a combination of trastuzumab and capecitabine with or without pertuzumab in patients with HER2-positive metastatic breast cancer (PHEREXA). https:// clinicaltrials.gov/ct2/show/NCT01026142. Accessed April 2, 2015.

32. ClinicalTrials.gov: A study of pertuzumab in combination with trastuzumab plus an aromatase inhibitor in patients with hormone receptor-positive, metastatic HER2-positive breast cancer. https://www.clinicaltrials.gov/ct2/show/NCT01491737. Accessed April 2, 2015.

33. Japan Breast Cancer Research Group: A study of eribulin in combination with trastuzumab and pertuzumab for advanced or recurrent human epidermal growth factor receptor 2 (HER2)-positive breast cancer (JBCRG-M03). http://www.jbcrg. jp/en/clinicaltrials/detail.php?id=32. Accessed April 2, 2015.

34. Japan Pharmaceutical Information Center: A post-marketing clinical study to evaluate the efficacy and safety of pertuzumab combined with trastuzumab and docetaxel as first line treatment in HER2- positive inoperable or recurrent breast cancer. http://www.clinicaltrials.jp/user/showCteDetailE. jsp?japicId=JapicCTI-132321. Accessed April 2, 2015.
35. ClinicalTrials.gov: A study of pertuzumab in addition to chemotherapy and Herceptin (trastuzumab) as adjuvant therapy in patients with HER2-positive primary breast cancer. https:// www.clinicaltrials.gov/ct2/show/study/NCT01358877. Accessed April 2, 2015

36. ClinicalTrials.gov: A Study of Kadcyla (trastuzumab emtansine) plus Perjeta (pertuzumab) following anthracyclines in comparison with Herceptin (trastuzumab) plus Perjeta and a taxane following anthracyclines as adjuvant therapy in patients with operable HER2-positive primary breast cancer. https://clinicaltrials.gov/ ct2/show/study/NCT01966471. Accessed April 2, 2015.

37. Verma S, Miles D, Gianni L, Krop IE, Welslau M, Baselga J, Pegram M, Oh DY, Diéras V, Guardino E, et al; EMILIA Study Group: Trastuzumab emtansine for HER2-positive advanced breast cancer. N Engl J Med 367: 1783-1791, 2012. 